\title{
Validation of the angular measurements of a new inertial-measurement-unit based rehabilitation system: comparison with state-of-the-art gait analysis
}

\author{
Alberto Leardini", Giada Lullini, Sandro Giannini, Lisa Berti, Maurizio Ortolani and Paolo Caravaggi
}

\begin{abstract}
Background: Several rehabilitation systems based on inertial measurement units (IMU) are entering the market for the control of exercises and to measure performance progression, particularly for recovery after lower limb orthopaedic treatments. IMU are easy to wear also by the patient alone, but the extent to which IMU's malpositioning in routine use can affect the accuracy of the measurements is not known. A new such system (Riablo ${ }^{\text {TM }}$, CoRehab, Trento, Italy), using audio-visual biofeedback based on videogames, was assessed against state-of-the-art gait analysis as the gold standard.
\end{abstract}

Methods: The sensitivity of the system to errors in the IMU's position and orientation was measured in 5 healthy subjects performing two hip joint motion exercises. Root mean square deviation was used to assess differences in the system's kinematic output between the erroneous and correct IMU position and orientation.

In order to estimate the system's accuracy, thorax and knee joint motion of 17 healthy subjects were tracked during the execution of standard rehabilitation tasks and compared with the corresponding measurements obtained with an established gait protocol using stereophotogrammetry.

Results: A maximum mean error of $3.1 \pm 1.8$ deg and $1.9 \pm 0.8$ deg from the angle trajectory with correct IMU position was recorded respectively in the medio-lateral malposition and frontal-plane misalignment tests. Across the standard rehabilitation tasks, the mean distance between the IMU and gait analysis systems was on average smaller than $5^{\circ}$.

Conclusions: These findings showed that the tested IMU based system has the necessary accuracy to be safely utilized in rehabilitation programs after orthopaedic treatments of the lower limb.

Keywords: Inertial measurement unit, Gait analysis, Rehabilitation, Knee, Hip, Thorax, Joint flexion, Audio-visual bio-feedback, Video-games

\section{Background}

Biofeedback has been used extensively in physical medicine and rehabilitation of human joints to facilitate recovery to normal function after injury and treatments [1]. Audio and visual feedbacks are intended to encourage patients to perform rehabilitation exercises with more attention, more accurately, and more frequently by adding entertainment to the execution of physical exercises. The signals on the position and orientation of the body segments involved in the movement exercise should provide

* Correspondence: leardini@ior.it

Movement Analysis Laboratory, Istituto Ortopedico Rizzoli, Bologna, Italy users with valuable feedback on the quality of their performance. This can be displayed in the basic form of numbers (direct inclinations or joint angles, general scores, etc.), geometrical entities or simple bar plots [2], up to complete immersive virtual environments typical of video-games [3-7].

Since manual and physical-exercise based physiotherapy provided in standard rehabilitation centres entails great expenses and resources, the use of self-administered training systems, which can be used at the patient's home, is being investigated $[8,9]$. These modern rehabilitation systems are highly portable, easy to use, and with a 
friendly graphical restitution, which is expected to facilitate the effective execution of standard and novel rehabilitation programs. Most of such systems are based on relatively low-cost inertial measurement units (IMU), which have been shown to be robust, small, and light to be worn on relevant body segments $[1,10,11]$. Typical target patients are those recovering from lower limb injury or joint reconstructions, these being usually adults keen to perform physical exercises at home $[12,13]$. While, on the one hand, a home-based rehabilitation program offers several advantages in terms of costs involved and convenience for the patient [14], on the other it is more subjected to human error that may hinder the correct application of the protocol and thus decrease its value.

Recently, a new such rehabilitation system has been developed and initially configured for the functional recovery of the lower limb joints. However, incorrect positioning of the IMU on the body segments in unsupervised utilization can hinder the system's performance, therefore its sensitivity in tracking joint rotations to known IMU's malposition and in standard end-user settings must be assessed. The aim of this study was to assess the system's reliability and accuracy during standard physical exercises using stereophotogrammetry as gold-standard.

\section{Methods}

\section{The IMU based rehabilitation system}

The Riablo $^{\mathrm{TM}}$ (CoRehab, Trento, Italy) is an adaptive system, comprised of several IMU connected wirelessly to a computer, developed to enhance standard rehabilitation programs by guiding the user in performing prescribed physical exercises through a video interface. The IMU used weighs 20 grams, is based on the wireless Bluetooth ${ }^{\mathrm{Tm}}$ communication protocol, and works at a sampling frequency of $50 \mathrm{~Hz}$. Nine degrees of freedom are provided by the following sensors: a 3D accelerometer at $\pm 2 \mathrm{~g}$ full-scale, a 3D gyroscope at $\pm 2000 \mathrm{dps}$ fullscale, and a $3 \mathrm{D}$ magnetometer at $\pm 1000 \mu \mathrm{T}$ full-scale.
The IMU sensors are calibrated at the factory before delivery.

Simple videogames provide audio and visual feedbacks to guide the subject in performing the rehabilitation exercises while wearing light IMU in a number of body segments. The units are self-worn on the frontal aspect of body segments via elastics bands. For the present study, shanks, thighs and thorax were instrumented to track knee and hip joint motion and the thorax inclination during the exercises (Figure 1).

The rotation angles are computed through a proprietary algorithm based on the Kalman filter theory [15]. Accordingly, a different weight is given to the position and orientation signals from the accelerometer $\left(\mathrm{k}_{\mathrm{a}}\right)$, the gyroscope $\left(\mathrm{k}_{\mathrm{g}}\right)$ and the magnetometer $\left(\mathrm{k}_{\mathrm{m}}\right)$, so that the sum $k_{a}+k_{g}+k_{m}$ is equal to 1 . The weighted collected signals are fused to provide a measure of the overall spatial orientation (pitch, roll, yaw) for each IMU.

A software calibration algorithm removes any offset associated to initial misalignments, typically due to IMU malpositioning and/or to the body segment peculiar shape. On screen instructions and recommendations help the user to limit the former as much as possible. Simple images show the user how to wear the elastic bands appropriately on the body segments, and to place the IMUs in the correct pouches according to color- and numerical- codes. A static calibration, which entails the user to maintain a double-leg up-right posture for a few seconds, is required to measure the neutral joint position between IMUs, according to standard angle calculation. This is assumed to be the initial offset to be used then in each dynamic exercise.

\section{IMU sensitivity}

The effects of different combinations of $\mathrm{k}$ weights on the kinematic-output were evaluated in a knee flexionextension exercise performed by one subject. For the optimal triplet of $\mathrm{k}$ weights, the system sensitivity to IMU malposition was assessed via two tests. One test

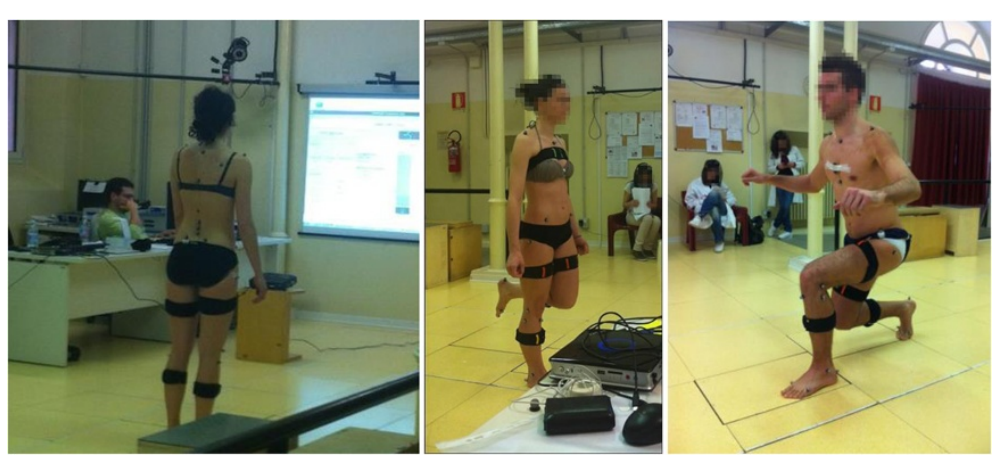

Figure 1 Pictures of the data collection. Two volunteers instrumented with IMU and markers for gait analysis during data collection in the gait laboratory; up-right posture (left), knee flexion against gravity (centre), lunge (right) are shown. 


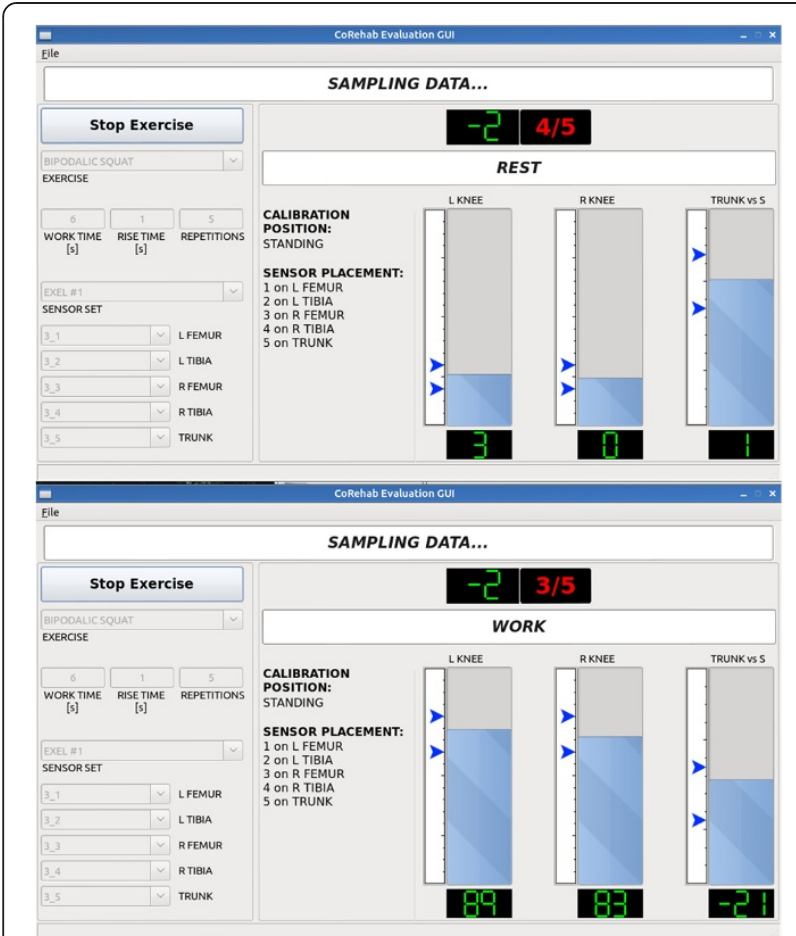

Figure 2 Screenshots of the visual feedback. Exemplary screenshots of the special interface used as visual feedback during the rest (top) and work (bottom) phases of the squat exercise. Users can monitor in real-time the knee (L KNEE and R KNEE) and thorax (TRUNK vs S, lunge and squat exercises only) flexion angles via bar plots and numerical values, and the desired target range by the position of the blue arrows. They can also have access (top of the screen) to the countdown (in green) and the repetition (red). aimed at assessing the effects on the calculated joint rotations for three frontal-plane orientations of the IMU $\left(0^{\circ}\right.$ correct; $-15^{\circ}$ and $\left.+15^{\circ}\right)$ within the elastic band in a hip abduction/adduction exercise (target abduction angle $=35^{\circ}$ ). Another test aimed at assessing the effects on the calculated joint rotations due to three medio-lateral positions of the IMU (correct, $-7 \mathrm{~cm}$ and $+7 \mathrm{~cm}$ ) in a hip flexion/extension exercise (target flexion angle $=90^{\circ}$ ). Both tests were performed by five healthy male subjects (25-35 years; $68-80 \mathrm{~kg} ; 165-190 \mathrm{~cm}$ ) each wearing three sets of IMU on the leg and thorax, for the three different configurations to be tested simultaneously. Root mean square deviation (RMSD) of the rotation trajectories over exercise duration in relation to those in the optimal IMU position/ orientation was used to estimate the system's sensitivity to IMU malpositioning.

\section{System accuracy}

The system accuracy in standard simulated rehabilitation activities was also tested in 17 healthy young adults (10 men, 7 women; age $26.3 \pm 3.8$ years; height $176.1 \pm 8.4 \mathrm{~cm}$; weight $69.6 \pm 11.8 \mathrm{~kg}$; BMI $22.3 \pm 2.3$ ), who volunteered for the study (Figure 1). The subjects were first instructed on how to wear the five elastic bands and relevant IMUs on the two thighs, shanks and on the thorax. These instructions were meant to simulate the initial training with the therapist as to make the user autonomous in operating the system. The following four exercises, typical of many rehabilitation programs for the knee joint, were performed on both left and right leg: lunge; knee flexion against gravity in single leg up-right posture; knee extension against gravity from the chair, and squatting. Five repetitions were recorded for each subject performing each exercise. The

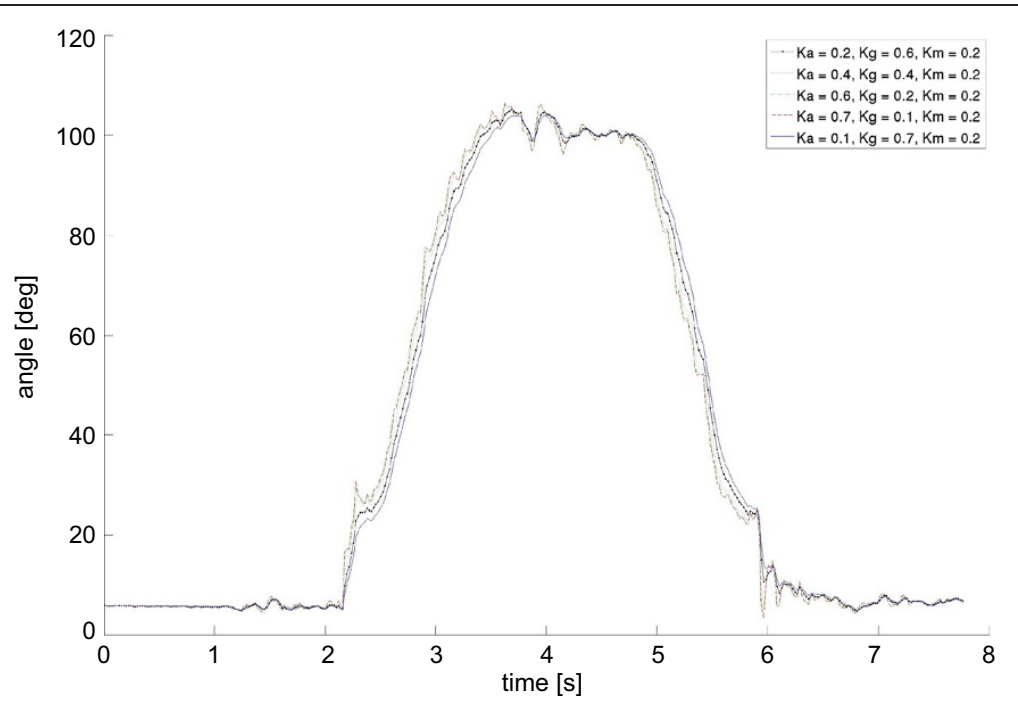

Figure 3 Kalman filter weights. Superimposition of flexion angle trajectories in a knee flexion against gravity exercise for different combinations of Kalman filter weights. Where $\mathrm{k}_{\mathrm{a}}, \mathrm{k}_{\mathrm{m}}$ and $\mathrm{k}_{\mathrm{g}}$ are the filter's weights of the accelerometer, magnetometer and gyroscope respectively. 
entire procedure, from IMU mounting to complete data collection, was repeated two times per each of the 17 subjects. In addition, up-right static posture was collected. The overall quality of the exercise was assessed through the analysis of knee joint rotations and thorax orientation, as recorded by the five IMU. In particular, in each exercise the targeted range of knee joint flexion was tracked by the thigh and shank IMU. For the lunge and squat only, as recommended by the specialists, the sagittal-plane inclination of the thorax was also tracked by the corresponding IMU. The IMU calculated angles, along with the targeted range, were displayed to the subject in real-time via a simple visual interface (Figure 2). The sequence of exercises, the target range of motion, and the rest time periods were also configured in the system and displayed to the user.

Simultaneously, three-dimensional rotations of the knee and thorax were measured via standard gait analysis (GA) system. Before starting the data collection, spherical $15-\mathrm{mm}$ reflective markers were located on the lower limbs, pelvis and thorax according to validated protocols [16,17], and tracked at $100 \mathrm{~Hz}$ during the exercise via an 8TV-camera stereophotogrammetric system (Vicon motion systems, UK). These markers established anatomical-based
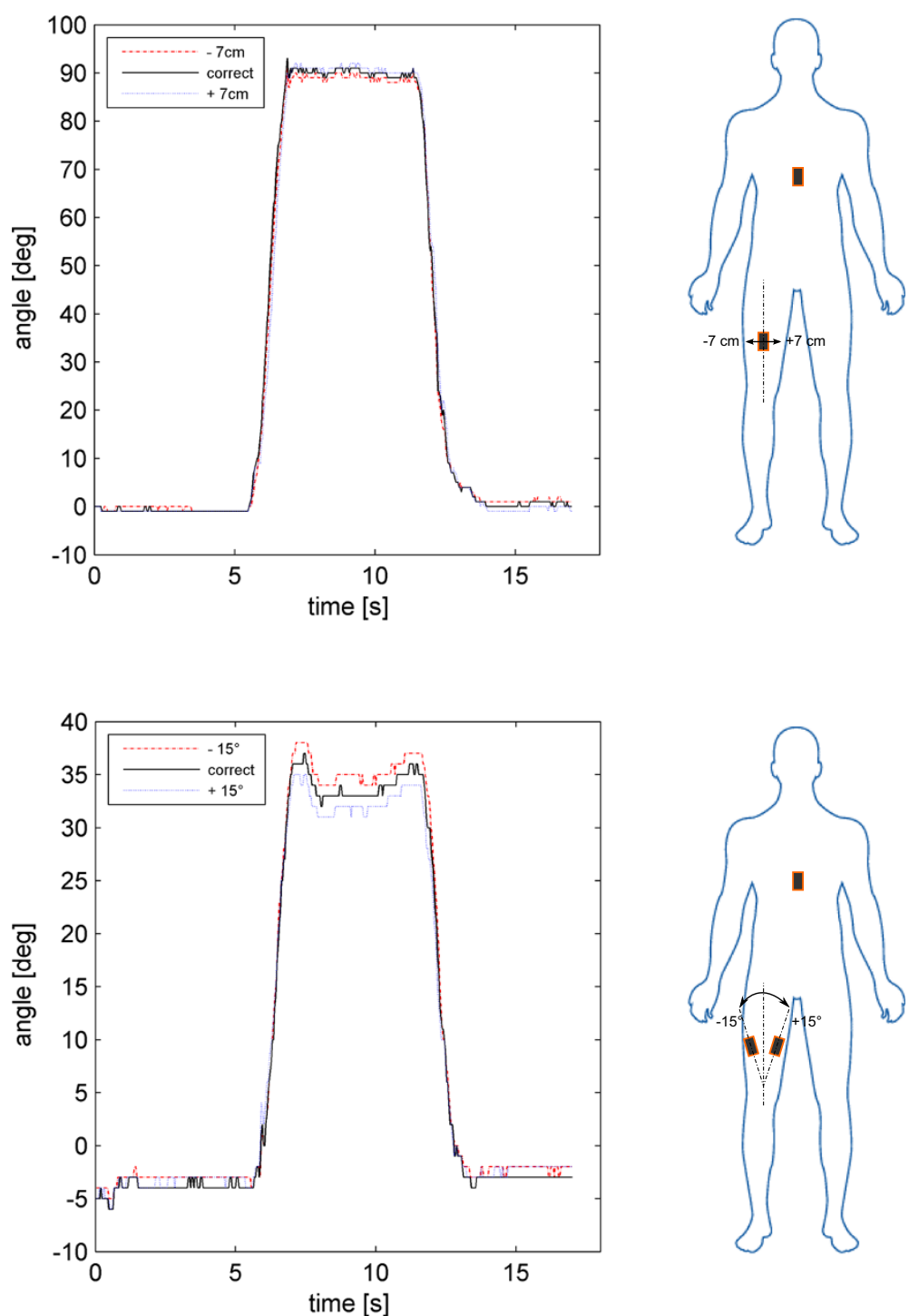

Figure 4 System sensitivity to IMU malposition. Top, superimposition of the kinematic trajectories [deg] from three pairs of IMU for a hip flexion/extension exercise in one subject. The three pairs of IMU were worn simultaneously on the subject's leg and thorax, each in one of the three positions of $-7 \mathrm{~cm}, 0 \mathrm{~cm}$, and $+7 \mathrm{~cm}$ with respect to the correct medio-lateral position. Bottom, superimposition of the kinematic trajectories [deg] from three pairs of IMU for a hip abduction exercise in one subject. The three pairs of IMU were worn simultaneously on the subject's leg and thorax, each in one of the three orientations of $-15^{\circ}, 0^{\circ}$ and $15^{\circ}$ with respect to the correct frontal-plane orientation. 
reference frames, from which knee flexion/extension and thorax inclination in the sagittal plane were determined according to international recommendations [18]. Motion of the thigh with respect to the shank, and of the thorax with respect to the laboratory in the sagittal plane only (i.e. flexion), were used as gold-standard for the corresponding IMU measurements. Synchronisation between IMU and GA measurements was achieved a-posteriori from visual inspection of the rotation patterns.

\section{Results}

\section{IMU sensitivity}

The optimal triplet of $\mathrm{k}$ weights ensuring a good compromise between output-signal smoothness and high responsiveness was: $\mathrm{k}_{\mathrm{a}}=0.2 ; \mathrm{k}_{\mathrm{g}}=0.6$, and $\mathrm{k}_{\mathrm{m}}=0.2$ (Figure 3). With this triplet of weights for the Kalman filter, the sensitivity of the system to IMU malpositioning was calculated. In the medio-lateral IMU configurations test (Figure 4, top), the RMSD of the output rotation trajectories in the -7 and $+7 \mathrm{~cm}$ configuration in relation to the optimal IMU position was, respectively, $2.1 \pm 1.5 \mathrm{deg}$ and $3.1 \pm 1.8 \mathrm{deg}$ across the 5 subjects. In the frontalplane test (Figure 4, bottom), the RMSD of the output rotation trajectories in the -15 and +15 deg configuration in relation to the optimal IMU orientation was, respectively, $1.3 \pm 0.6 \mathrm{deg}$ and $1.9 \pm 0.8 \mathrm{deg}$ across the 5 subjects.

\section{System accuracy}

The GA and IMU calculated knee flexion and thorax inclination angles were superimposed for each exercise (see Figure 5). In the squat exercise (bottom, Figure 5), five repetitions of about $80^{\circ}$ knee flexion were performed in about 70 seconds, along with thorax motion in the sagittal plane between $0^{\circ}$ and $45^{\circ}$. In the knee, a small
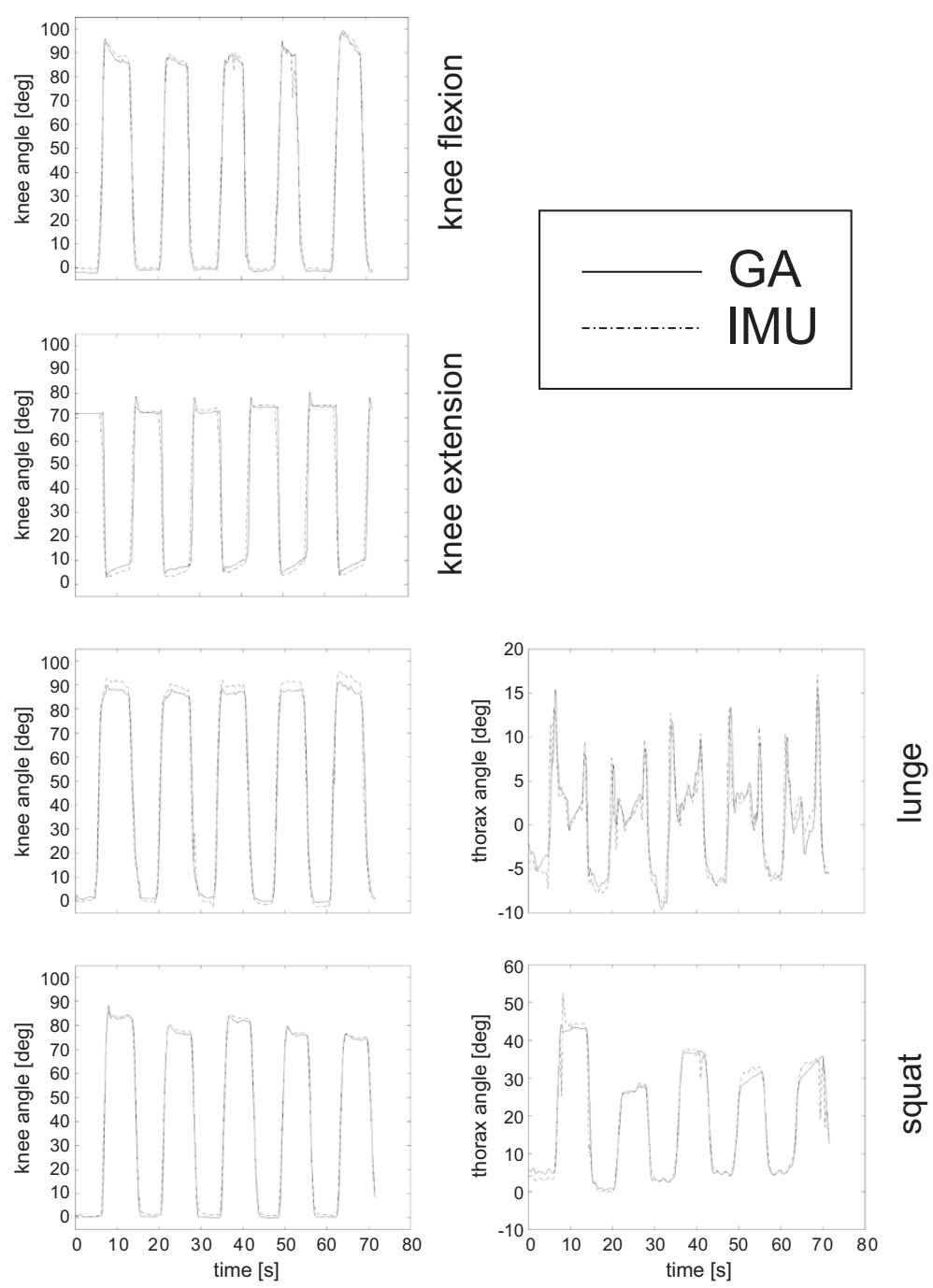

Figure 5 Superimposition of GA and IMU measurements of knee and thorax flexion patterns. Superimposition of IMU and corresponding GA flexion angles of the knee (left column) and thorax (right column) over each exercise (rows) in a typical subject. 
Table 1 Knee error

\begin{tabular}{lcccc}
\hline Exercise: & \multicolumn{3}{c}{ Knee flexion } \\
\cline { 2 - 5 } & Target ROM & Mean distance & MIN mean dist. & MAX mean dist. \\
\hline Knee flexion & $0-95$ & $3.9 \pm 0.7$ & 2.2 & 6.1 \\
Knee extension & $0-90$ & $3.8 \pm 0.8$ & 1.8 & 5.9 \\
Lunge & $0-100$ & $4.5 \pm 1.3$ & 2.0 & 7.9 \\
Squat & $0-100$ & $5.0 \pm 1.2$ & 2.4 & 8.3 \\
\hline
\end{tabular}

Differences between IMU and GA for knee flexion angles [deg] over the 17 subjects analyzed.

bias can be observed in knee flexion and squat exercise, and a little larger range of flexion is calculated by the IMU system in knee extension and lunge exercises. With the exclusion of a few spikes from the IMU, thorax motion compared well across the exercises.

The mean distance between the joint rotation angles recorded by GA and IMU systems was evaluated for each subject and each exercise and averaged over the subjects (Tables 1 and 2). Mean rotation difference was smaller than 5 and 3 degrees respectively for knee flexion and for thorax inclination. The best (minimum distance) and the worst (maximum distance) measure for each exercise is also reported.

\section{Discussion}

The Riablo system was developed to enhance physical rehabilitation by motivating the user in the execution of prescribed exercises either under the supervision of the physiotherapist or independently at home. Simple videogames provide audio and visual feedback according to the orientation and movement of light IMU worn on relevant body segments. Type and difficulty of the videogames were designed by specialists to address different rehabilitation needs.

New measurement units for human segment and joint motion should be validated before being introduced into the clinical setting. Recently, this has been performed for a novel motion tracking systems originally designed for video-games [19]. Several original IMUbased techniques to track lower limb joints motion have been proposed $[10,11,20,21]$, but only a few have been validated using stereophotogrammetry as the gold-standard [19,22-28], as performed in the present study. As expected, the knee flexion angle was found to be the best to be estimated by the IMU among the three rotations [25].
In the present study, the sensitivity of the system to errors in IMU positioning in measuring joint angle trajectories appeared to be acceptable in the scenario of typical lower limb rehabilitation programs. While only a few erroneous IMU configurations were tested in this study, and no combinations of mal-orientation and malposition were evaluated, the extent of erroneous malpositioning in routine usage is limited by the conforming shape of the pouch carrying the IMU in the elastic band. Moreover, absolute IMU deviations from the correct vertical alignment larger than $15^{\circ}$ result in the calibration process to fail and a warning message being displayed to the user to correct the IMU position. As for the system's accuracy, the knee joint angles calculated by the IMUs compared very well with those obtained from gait analysis based on stereophotogrammetry, though these IMUs were self-worn by the subjects as in the actual rehabilitation settings. Similarly, the thorax flexion was found to be well estimated by the corresponding IMU, as already reported in the relevant literature $[22,29]$.

It should be highlighted that the high quality/resolution of the videogames, normally used to guide the users to perform the exercises for this system, would have required a relatively low data collection sampling rate. Therefore, for the present validation study, special audio and visual feedbacks (Figure 2) were used to allow the IMU system's sampling frequency to better match the $100 \mathrm{~Hz}$ of the stereophotogrammetric system. Deviations from the targeted degrees of knee flexion may be considered acceptable for these rehabilitation exercises to be safe to the patient.

While no major differences are expected to be found in patients after standard orthopaedic treatments of the lower limb joints, the present study is limited by the population of young and healthy subjects analysed. The influence of severe knee deformities on the calculated

Table 2 Thorax error

\begin{tabular}{lcccc}
\hline Exercise: & \multicolumn{3}{c}{ Thorax inclination } \\
\cline { 2 - 5 } & Target ROM & Mean distance & MIN mean dist. & MAX mean dist. \\
\hline Lunge & $0-25$ & $1.6 \pm 0.6$ & 0.6 & 3.8 \\
Squat & $0-45$ & $2.7 \pm 2.1$ & 0.6 & 7.5 \\
\hline
\end{tabular}

Differences between IMU and GA for thorax inclination [deg] over the 17 subjects analyzed. 
joint flexion/extension trajectories and the accuracy in tracking other segments and joints should be investigated separately in future studies. Finally, the gait analysis technique adopted might have its own limitations and different definitions, but it is among the most complete and validated, designed according to international standards in biomechanics.

\section{Conclusions}

The present work investigated the sensitivity and accuracy of a modern rehabilitation system, in particular the angular measurements at the knee and thorax were compared with the corresponding measurements from state-of-theart gait analysis. The results showed that the IMU based system has small errors in measuring joint rotations even in the present self-worn condition. The system appears therefore suitable to be used in routine rehabilitation of the lower limb joints, following orthopaedic treatment or during recovery from injury, also in a self-administered home setting.

\section{Competing interests}

The Institute of the Authors has received funding from the company marketing the Riablo system to cover the costs involved with the execution of this study.

\section{Authors' contributions}

AL conceived the study, coordinated data collection and analysis, and drafted the manuscript. GL contributed to the design of the study, helped to data collection, and to draft the manuscript. PC participated in the design of the study, helped in data analysis and in manuscript revision. LB participated in the design of the study and performed data interpretation. MO carried out the data collection and pre-analysis. SG participated in the design of the study and its coordination. All authors read and approved the final manuscript.

\section{Acknowledgements}

The Authors acknowledge CoRehab s.r.l. for their technical and economical contribution to this study.

Received: 6 May 2014 Accepted: 2 September 2014

Published: 11 September 2014

\section{References}

1. Giggins OM, Persson UM, Caulfield B: Biofeedback in rehabilitation. J Neuroeng Rehabil 2013, 10:60

2. McClelland J, Zeni J, Haley RM, Snyder-Mackler L: Functional and biomechanical outcomes after using biofeedback for retraining symmetrical movement patterns after total knee arthroplasty: a case report. J Orthop Sports PhysTher 2012, 42(2):135-144.

3. Taylor MJ, McCormick D, Shawis T, Impson R, Griffin M: Activity-promoting gaming systems in exercise and rehabilitation. J Rehabil Res Dev 2011, 48(10):1171-1186

4. Thompson D: Designing serious video games for health behavior change: current status and future directions. J Diabetes Sci Technol 2012, 6(4):807-811.

5. Primack BA, Carroll MV, McNamara M, Klem ML, King B, Rich M, Chan CW, Nayak S: Role of video games in improving health-related outcomes: a systematic review. Am J Prev Med 2012, 42(6):630-638.

6. Lewis GN, Rosie JA: Virtual reality games for movement rehabilitation in neurological conditions: how do we meet the needs and expectations of the users? Disabil Rehabil 2012, 34(22):1880-1886.

7. LeBlanc AG, Chaput JP, McFarlane A, Colley RC, Thivel D, Biddle SJ, Maddison R, Leatherdale ST, Tremblay MS: Active video games and health indicators in children and youth: a systematic review. PLoS One 2013, 8(6):e65351.
8. Faria C, Silva J, Campilho A: Rehab@home: a tool for home-based motor function rehabilitation. Disabil Rehabil Assist Technol 2013, Sep 26. [Epub ahead of print].

9. Prosperini L, Fortuna D, Giannì C, Leonardi L, Marchetti MR, Pozzilli C: Homebased balance training using the Wii balance board: a randomized, crossover pilot study in multiple sclerosis. Neurorehabil Neural Repair 2013, 27(6):516-525.

10. Fong DT, Chan YY: The use of wearable inertial motion sensors in human lower limb biomechanics studies: a systematic review. Sensors (Basel) 2010, 10(12):11556-11565.

11. Cuesta-Vargas Al, Galán-Mercant A, Williams JM: The use of inertial sensors system for human motion analysis. Phys Ther Rev 2010, 15(6):462-473.

12. Kruse LM, Gray B, Wright RW: Rehabilitation after anterior cruciate ligament reconstruction: a systematic review. J Bone Joint Surg Am 2012, 94(19):1737-1748.

13. Howells BE, Clark RA, Ardern CL, Bryant AL, Feller JA, Whitehead TS, Webster $\mathrm{KE}$ : The assessment of postural control and the influence of a secondary task in people with anterior cruciate ligament reconstructed knees using a Nintendo Wii Balance Board. Br J Sports Med 2013, 47:914-919.

14. Zheng $H$, Black ND, Harris ND: Position-sensing technologies for movement analysis in stroke rehabilitation. Med Biol Eng Comput 2005, 43(4):413-420.

15. Kalman RE: A new approach to linear filtering and prediction problems. J Basic Eng 1960, 82(1):35-45.

16. Leardini A, Sawacha Z, Paolini G, Ingrosso S, Nativo R, Benedetti MG: A new anatomically based protocol for gait analysis in children. Gait Posture 2007, 26(4):560-571.

17. Leardini A, Biagi F, Merlo A, Belvedere C, Benedetti MG: Multi-segment trunk kinematics during locomotion and elementary exercises. Clin Biomech (Bristol, Avon) 2011, 26(6):562-571.

18. Wu G, Siegler S, Allard P, Kirtley C, Leardini A, Rosenbaum D, Whittle M, D'Lima DD, Cristofolini L, Witte H, Schmid O, Stokes I: ISB recommendation on definitions of joint coordinate system of various joints for the reporting of human joint motion-part l: ankle, hip, and spine. J Biomech 2002, 35(4):543-548.

19. Bonnechère B, Jansen B, Salvia P, Bouzahouene H, Omelina L, Moiseev F, Sholukha V, Cornelis J, Rooze M, Van Sint JS: Validity and reliability of the Kinect within functional assessment activities: comparison with standard stereophotogrammetry. Gait Posture 2014, 39(1):593-598.

20. Favre J, Jolles BM, Aissaoui R, Aminian K: Ambulatory measurement of $3 D$ knee joint angle. J Biomech 2008, 41:1029-1035.

21. Cutti AG, Ferrari A, Garofalo P, Raggi M, Cappello A, Ferrari A: 'Outwalk': a protocol for clinical gait analysis based on inertial and magnetic sensors. Med Biol Eng Comput 2010, 48:17-25.

22. Picerno $P$, Cereatti A, Cappozzo A: Joint kinematics estimate using wearable inertial and magnetic sensing modules. Gait Posture 2008, 28(4):588-595.

23. Ferrari A, Cutti AG, Garofalo P, Raggi M, Heijboer M, Cappello A, Davalli A: First in vivo assessment of "Outwalk": a novel protocol for clinical gait analysis based on inertial and magnetic sensors. Med Biol Eng Comput 2010, 48(1):1-15.

24. Watanabe T, Saito H: Tests of wireless wearable sensor system in joint angle measurement of lower limbs. Conf Proc IEEE Eng Med Biol Soc 2011, 2011:5469-5472

25. Laudanski A, Brouwer B, Li Q: Measurement of lower limb joint kinematics using inertial sensors during stair ascent and descent in healthy older adults and stroke survivors. J Healthc Eng 2013, 4(4):555-576.

26. van den Noort JC, Ferrari A, Cutti AG, Becher JG, Harlaar J: Gait analysis in children with cerebral palsy via inertial and magnetic sensors. Med Biol Eng Comput 2013, 51(4):377-386.

27. Slajpah S, Kamnik R, Munih M: Kinematics based sensory fusion for wearable motion assessment in human walking. Comput Methods Programs Biomed 2014, 116(2):131-144.

28. Seel T, Raisch J, Schauer T: IMU-based joint angle measurement for gait analysis. Sensors (Basel) 2014, 14(4):6891-6909.

29. Plamondon A, Delisle A, Larue C, Brouillette D, McFadden D, Desjardins P, Larivière C: Evaluation of a hybrid system for three-dimensional measurement of trunk posture in motion. Appl Ergon 2007, 38(6):697-712.

\section{doi:10.1186/1743-0003-11-136}

Cite this article as: Leardini et al:: Validation of the angular measurements of a new inertial-measurement-unit based rehabilitation system: comparison with state-of-the-art gait analysis. Journal of NeuroEngineering and Rehabilitation 2014 11:136. 